\title{
Caenorhabditis elegans as a Test Organism for Detecting Soil Toxicity in Saudi Arabia
}

\author{
Saleh Alhewairini, ${ }^{1, *}$, Ian R Mellor ${ }^{2}$, Ian R Duce ${ }^{2}$ \\ ${ }^{1}$ The Agricultural and Veterinary College, Qassim University, Saudi Arabia \\ ${ }^{2}$ School of Life Sciences, University of Nottingham, UK
}

Copyright (C) 2016 by authors, all rights reserved. Authors agree that this article remains permanently open access under the terms of the Creative Commons Attribution License 4.0 International License

\begin{abstract}
The extensive and intensive use of many pesticides in agriculture has caused environmental pollution. Chemicals directly added to the soil, whether in greenhouses or in open fields can reach ground water, rivers and lakes by run off, where significant amounts may directly or indirectly affect human health. Therefore, interest has grown to establish appropriate, fast and inexpensive methods to assess the environmental impacts of chemicals, including pesticides, in soil. Soil organisms are important for soil fertility, and sustainability of soil ecosystems can be disrupted by soil toxicity. The main aim of this study is to establish a novel and convenient assay to investigate and quantify soil toxicity.
\end{abstract}

Keywords C.elegans, Oxamyl, Pharyngeal Pumping, Pesticides, Nematodes

\section{Introduction}

The monitoring of pollutants and other chemical residues in the environment is an important part of environmental protection. Due to their inherently toxic nature there is a public perception that pesticides pose a particular threat to the environment and therefore monitoring their levels is an important part of environmental monitoring. There are many sensitive methods using gas or liquid chromatography coupled to sensitive detectors such as mass spectrometers which are the basis for setting environmental standards by regulatory and legislative bodies [1]. The methods are laid down as international standards regulated by the International Standards Organization (ISO) [2]. Although these methods are accurate and precise they require a significant investment in equipment and trained personnel. It is also not always clear how they relate to toxic effects on organisms, therefore, biomarkers have been extensively used to assess environmental pollution. There are many reviews of the use of whole-organisms and molecular biomarkers for ecotoxicology including aquatic and soil ecosystems where pesticides might be expected to accumulate. A full review of this subject is beyond the scope of this research topic; however, a recent review advocates the use of Daphnia as part of routine environmental risk assessment of plant protection products [3].

Soil contains microflora and fauna which play an important role in sustainability and fertility and can be disrupted by soil contamination [4]. Thus, it is important to establish appropriate methods to assess toxicity of soil contaminants. Soil toxicity has been assessed using plants, bacteria, collembolans and earthworms [5]; unfortunately the most abundant group of soil invertebrates, namely nematodes are not given importance in soil toxicity studies. There are thought to be almost 500,000 species worldwide [6] and their activity plays an essential role for soil [7] as well as agricultural production and sustainability [8].

Caenorhabditis elegans has been successfully used as a test organism in several environmental studies to investigate soil toxicity [4, 9-15]. C.elegans was considered to be a suitable test organism as its cuticle allows uptake of water soluble materials such as dissolved metals [12].

Several endpoints such as changes in behaviour, lethality, gene expression, mortality and feeding have been examined in C.elegans to investigate toxicological effects of chemical compounds including heavy metals and pesticides [16-18].

Hoss et al. [4] used C.elegans as a test organism to test nine uncontaminated reference soils and 22 contaminated soils with different chemical properties by observing the growth, reproduction and fertility of C.elegans. They found out that in reference soils C.elegans showed a very low variability of growth and $100 \%$ fertility whereas the contaminated soils showed clear toxic effects on C.elegans. More recently Anbalagan et al. [15] tested a range of pesticides on 24 GFP stress-gene reporter strains of C.elegans and discovered that both the organochlorine endosulfan and the organophosphate dichlorvos were capable of inducing stress genes but with different patterns of gene induction. They also demonstrated significant toxicity from uncharacterised organic contamination in agricultural soil from south east Spain. 
It has been shown that pharyngeal pumping in C.elegans is sensitive to a range of compounds [16, 19] including pesticides and the aim of the work presented in this research paper was to test the utility of the assay for assessing toxicants in field samples.

In Saudi Arabia, there is little information and no database available regarding soil toxicity due to the extensive use of agrochemicals either in greenhouses or in open fields. To address this problem a study was carried out to collect soil samples from intensively cultivated greenhouses and soil from adjacent uncultivated sites and to assess whether soil pore water from these samples produced a change in pharyngeal pumping.

\section{Materials and Methods}

Forty six soil samples from greenhouses and 30 control samples were collected from 15 different farms in Saudi Arabia in September 2012. Some farms were air conditioned greenhouses (which tend to be more intensively cultivated), whilst some were only normal (non-air conditioned) and there were some which had both facilities. From each farm, control soil samples were collected from outside of the greenhouse approximately 10 metres away (depending on the layout of the farm). A sample of irrigation water was also tested for $\mathrm{pH}$ and conductivity.

Electrical conductivity (EC) and $\mathrm{pH}$ was directly measured by inserting an EC electrode or a $\mathrm{pH}$ electrode into the suspension. Water samples were directly collected from well water of each farm. EC and $\mathrm{pH}$ of water samples were also measured. Assessment of the organic content of soil samples were done following the method of Walkley and Black [20]. Each farm was allotted a letter from $\mathrm{A}$ to $\mathrm{O}$ and a numerical sub-script indicated the sample number or replicate. This step was taken to conceal the identity of the farm.

Soil extraction described by Anbalagan et al. [14] was followed. Initial experiments were carried out to establish whether the extraction method could elute known toxicants added to a soil sample for detection in the pharyngeal pumping assay. A simulated soil sample was prepared from 90\% horticultural sand and 10\% Cactus Compost (w/w) purchased from Wheatcroft's Garden Centre, Nottingham, UK. $20 \mathrm{~g}$ of the mixture was transferred to a Petri dish, and allowed to dry. $5 \mathrm{ml}$ of distilled water (control) or $5 \mathrm{ml}$ of either $300 \mathrm{ppm}$ or $1200 \mathrm{ppm}$ oxamyl were sprayed onto the simulated soil samples in Petri dishes. The dishes were left overnight to dry at room temperature $\left(20^{\circ} \mathrm{C}\right)$. The same method was then used to elute the simulated samples and the samples collected from greenhouses as described below: $50 \%(\mathrm{v} / \mathrm{w})$ of distilled water was added to each soil sample ( $50 \mathrm{ml}$ distilled water plus $100 \mathrm{~g}$ of soil sample) and mixed in a beaker. The suspension was left at $4^{\circ} \mathrm{C}$ overnight to enable soluble material to leach out. The content of each beaker was transferred to a $20 \mathrm{ml}$ syringe plugged with glass wool mounted in a $50 \mathrm{ml}$ centrifuge tube. Each $50 \mathrm{ml}$ tube was centrifuged at $5000 \mathrm{rpm}$ for 5 minutes. The $20 \mathrm{ml}$ syringe containing the soil sample was removed. The eluate was centrifuged again and the clear solution was transferred to a $15 \mathrm{ml}$ tube using a pasture pipette. The extracted water was initially tested as extracted (100\%), and diluted as necessary depending upon the level of activity. $750 \mu$ l of extracted water was transferred to labelled 6 well plates $+750 \mu \mathrm{l}$ $\mathrm{K}$-medium and N2 (wild-type) C.elegans. Plates were incubated at $20^{\circ} \mathrm{C}$ for 1 hour and 24 hours. After 1 hour or 24 hours a $200 \mu \mathrm{l}$ sample was taken from each well using a micropipette and transferred to small plates containing normal nematode growth medium (NGM) (Sodium chloride (3.0g/l) Agar (17.0g/1) Peptone (2.5g/l) Cholesterol (5mg/l) Distilled water $(975 \mathrm{ml})$ Calcium chloride $(1 \mathrm{mM})$ Magnesium sulphate $(1 \mathrm{mM})$ Potassium phosphate $(2 \mathrm{mM})$ ) without bacteria. Individual C.elegans were isolated using a flamed platinum wire pick and transferred to a small plate containing NGM without bacteria. Pharyngeal pumping rate was counted manually using a stop watch by direct observation under a microscope and expressed as pumps/minute. For each treatment the pumping rate of 5 worms was determined and expressed as the mean pumping rate $\pm \mathrm{SEM}$.

Soil samples showing activity in the C.elegans pharyngeal pumping assay were subject to chemical analysis. $20 \mathrm{ml}$ of soil eluate was made up to $500 \mathrm{ml}$ with distilled water in clean glass bottles provided by ALcontrol Laboratories and transported in ice cooled containers to the analytical laboratories. They were analysed using an Agilent 6890 gas chromatography system with coupled Agilent 5973 Mass Spectrometer with an ISO 1725 accredited method for the detection of organophosphorus and organochlorine pesticides and triazine herbicides, with a limit of detection of $0.01 \mu \mathrm{g} / 1$.

\section{Results}

An initial pilot study was carried out with a laboratory prepared soil sample treated with a known concentration of the commonly used soil carbamate nematicide, oxamyl. The aim of this experiment was to establish whether the soil extraction process would release a toxicant from soil pore water with a known effect on pharyngeal pumping. The results are shown in figure 1 and show that soil samples eluted after treatment with either $300 \mathrm{ppm}$ or $1200 \mathrm{ppm}$ oxamyl in water produced a significant inhibition of pharyngeal pumping compared with control samples treated with water. 


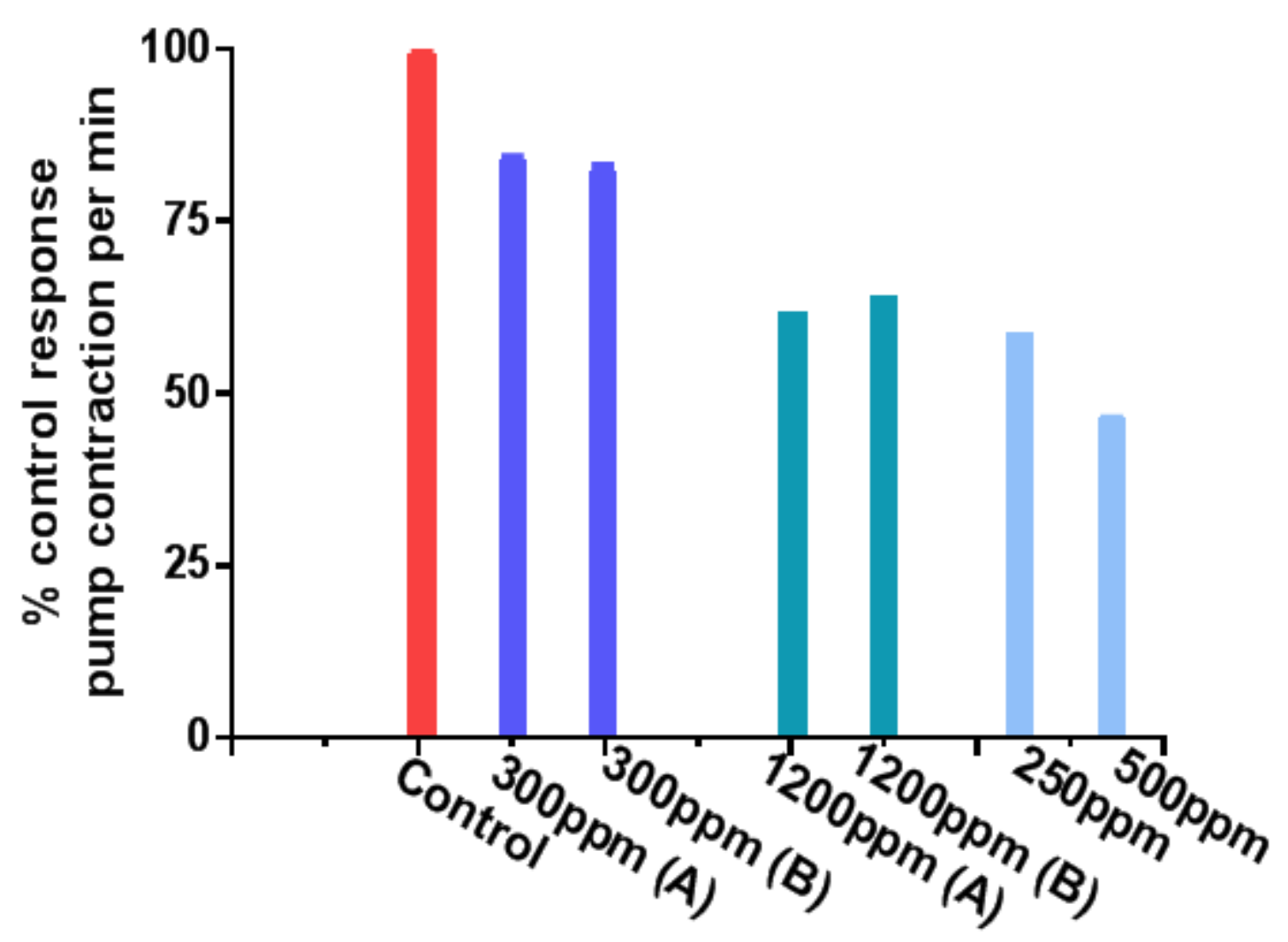

Figure 1. Comparison of the effects of soil eluate after spraying with water (control) or oxamyl (300 and 1200ppm) on the rate of pharyngeal pumping in N2 C.elegans expressed as \% of the control pumping rate in K-medium without eluate. Each bar shows the mean \pm SEM of 5 replicates. A and B indicate replicated samples. For comparison the pumping rates of worms treated directly with 250 and 500ppm of oxamyl (without soil elution) are shown.

The physico-chemical properties of the soil samples were tested. The water $\mathrm{pH}$ in all farms was slightly alkaline and the conductivity varied showing different mineral content of the water. The soil $\mathrm{pH}$ was a mostly consistent alkaline pattern with $\mathrm{pH}$ between 8 and 9 . The conductivity of the soil suspensions from the greenhouses varied with air conditioned greenhouses usually having higher conductivity and with control samples often having much lower conductivity presumably due to the fertilizer and other mineral content added to the cultivated soils in the greenhouses. Organic matter also varied considerably with some soils having very low levels of organic content or carbon. The final soil eluates had a very consistent $\mathrm{pH}$ between 7.3 and 8.1.

The eluate from each sample was tested, without dilution, on pharyngeal pumping. The control samples from outside of the greenhouse were averaged to provide a single average for each site. C.elegans were incubated for 1 hour and 24 hours before measuring pharyngeal pumping.

For each greenhouse sample the reduction in pharyngeal pumping was calculated as a percent reduction relative to its control. The movements of C.elegans were also observed.

There were clear qualitative and quantitative differences between the effects of the eluates from different farms. In most cases, the differences between air-conditioned and normal greenhouses was relatively small but there was evidence from farms A, D and I that the soil from air conditioned greenhouses was more toxic than from normal greenhouses, but in the case of farm $\mathrm{A}$ and $\mathrm{D}$ the differences were less marked when C.elegans were incubated for 24 hours than for 1 hour.

The most striking effect was seen in farm $\mathrm{M}$ where the eluate had profound effects on both pharyngeal movement and locomotion and inhibition of pharyngeal pumping and paralysis was consistent at 1 hour and 24 hours.

The toxicity of the eluate from farm $\mathrm{M}$ was further studied by carrying out a dose-response assay by diluting the eluate from $100 \%$ to $50 \%, 25 \%, 10 \% 5 \%$ and $1 \%$ and measuring pharyngeal pumping at each concentration. The dose-response curves are shown in figure 2 . In each case there is a clear dose-inhibition relationship and the most toxic samples from site M1-1 had a maximum inhibition of pumping of around $60 \%$ which was consistent after 1 and 24 hours incubation. There was little difference in the dose-inhibition curves after incubating C.elegans for 1 or 24 hours in the eluate from M1-1 but the curves from the other sites suggest that the more dilute samples were more effective after 1 hour than 24 hours of incubation. 

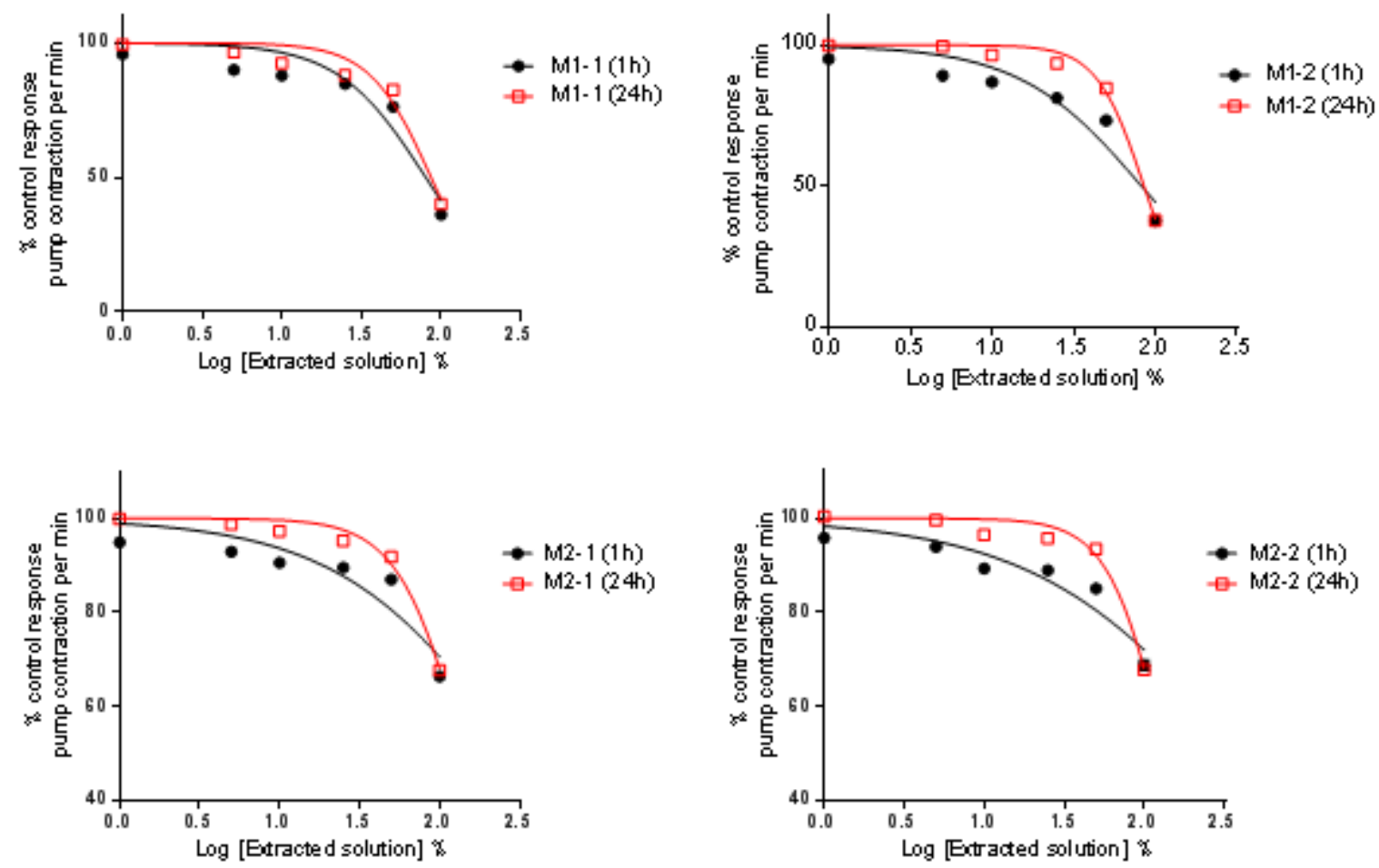

Figure 2. Comparison of the effects of the extracted solution of M1-1, M1-2, M2-1 and M2-2 after 1 hour \& 24 hours incubation on the rate of pharyngeal pumping in N2 C.elegans expressed as \% of the control pumping rate in K-medium without eluate. Each point is the mean \pm SEM of 5 replicates, but in most cases the error bars are smaller than the symbols used. The lines were fitted using a non-linear regression in GraphPad Prism 6 with the maximum plateau as $100 \%$ and the minimum as $0 \%$.

The pharyngeal pumping assay is therefore capable of detecting toxicants in field samples and is able to discriminate between the toxicity of soils from different sites and therefore can serve as a useful biomarker in initial monitoring. It seems likely from data presented that the eluate from some of these sites contains one or more of the neurotoxic nematicides which are heavily used in intensive horticulture in Saudi Arabia, particularly to control plant parasitic nematodes. This is supported by personal knowledge that greenhouses in Saudi Arabia receive extensive and poorly recorded or regulated administration of insecticides and nematicides. It is unknown from these experiments what the toxicants might be or at what concentration they might be present. We can, however, make an estimate by comparison of the dose-response curves. The apparent $\mathrm{IC}_{50}$ is about $80 \%$ of the eluate. If we compare that with values for a widely used nematicide, oxamyl (figure 1), the inhibition is equivalent to that caused by ca. $350 \mathrm{ppm}$ oxamyl. A reasonable estimate would therefore be that the eluate contains toxicant or toxicants with the equivalent potency of 300-400ppm oxamyl. We decided on that basis to submit the eluate for chemical analysis by Alcontrol laboratories (Hawarden, Cheshire, UK). 12 eluates of samples taken from farms M (M 1-1, M 1-2, M 2-1 M 2-2, M.C 1 and M.C 2) and H (H 1-1, H1-2, H 2-1 H 2-2, H.C 1 and H.C 2) were diluted by taking $20 \mathrm{ml}$ of the eluate and adding $480 \mathrm{ml}$ double distilled water in glass bottles supplied by the analytical lab. The samples were transported in cooled containers and analysed by GCMS according to ISO 17025 for 28 different organochlorine and organophosphate insecticides.

Our estimate of the effective concentration of the diluted material sent for analysis from the most potent samples, e.g. M1-1 would be in the order of $32 \mathrm{ppm}$ i.e. $32 \mathrm{mg} / 1$ and should therefore be well in excess of the limits of detection of the assay $(0.01 \mu \mathrm{g} / \mathrm{l})$.

Disappointingly, the 12 samples had no compounds above the limit of detection except malathion which had a level $<0.03 \mu \mathrm{g} / \mathrm{l}$ in all samples. As it is present in all samples, including the controls, we assume this is an artifact. The one positive contaminant detected was from sample M2-2 where azinphos-methyl was present at $0.09 \mu \mathrm{g} / \mathrm{l}$. Furthermore, the negative finding suggests that other pesticides and/or degradation products may account for the toxicity of the soil eluate samples.

\section{Discussion}

The heavy application of pesticides has been associated with the increase in food consumption and can result in contaminating sites close to or at a distance from the site of application. The potential for contamination by human activity such as misuse of pesticides and discarding of pesticide containers in conjunction with natural processes including rain and wind can result in chemical substances 
being leached from soil leading to contamination of rivers, lakes and sea. Therefore, the experiments here were designed to test the content of soil pore water eluted from soil samples collected from glasshouses in Saudi Arabia to establish whether the C.elegans pharyngeal pumping assay is a useful system to quantify the presence of environmental pollution due to pesticide application.

The pharyngeal pumping assay does seem to be able to differentiate the toxicity of soils from different farms, is relatively sensitive, cheap and straightforward to carry out and provides easily quantifiable data. It would be useful to carry out more pilot studies to establish how well it can detect the commonly used agrochemicals and mixtures of different chemicals. It is possible that its sensitivity may be restricted by access of toxicants to target sites in the pharynx, and although Sochova et al. [12] suggested C.elegans has a thin water-permeable cuticle covering its body, making it more sensitive to dissolved metals, it has been necessary in some experiments analyzing the effects of anthelmintics on pharyngeal pumping to use a cut preparation where the anterior of the organism is transected from the rest of the body to provide better access by drugs $[21,22]$. It may be useful to compare the sensitivity of the assay using intact C.elegans with a cut preparation to see if there is any difference in sensitivity. Pesticides vary in terms of their hydrophobicity which may also affect their movement across the C.elegans cuticle.

Sochova et al. [12] pointed out the need to consider other factors such as $\mathrm{pH}$, organic content and soil type, and the need to use a reference sample from all test sites which should have the same soil properties as the contaminated site. Carbon and organic content, $\mathrm{pH}$ and salinity of soil samples have been shown to influence degradation and solubility of many chemical compounds including heavy metals and pesticides. For example the $\mathrm{pH}$ has a significant role in enhancing solubility of heavy metals (e.g. $\mathrm{Pb}, \mathrm{Cd}$ and $\mathrm{Zn}$ ) in soil samples [23] where acidic conditions promote metal solubility. Another study by Hoss et al. [4] confirmed that at higher $\mathrm{pH}$, metals are also insoluble in the pore water phase. The Saudi Arabian soils tested here mostly had a high $\mathrm{pH}$ and salinity. It does not seem likely that the $\mathrm{pH}$ and salinity values seen in these experiments would affect C.elegans directly, as Khanna et al. [24] tested the sensitivity of C.elegans to both $\mathrm{pH}$ and salinity in aquatic media. They found that up to $15.49 \mathrm{~g} / 1 \mathrm{NaCl}(15490 \mathrm{ppm})$ in K-medium had no significant effects on lethality and was tolerated by C.elegans. They also highlighted that a $\mathrm{pH}$ range of 3.1 to 11.9 for 24 hours and 3.2 to 11.8 for 96 hours had no significant effects on the survival of C.elegnas and make the organism very suitable for soil monitoring. It was also notable that farm $\mathrm{H}$ had the lowest organic and carbon content and the soil had least effect on pharyngeal pumping. This may suggest that organic content is important in the retention of the toxicants; alternatively the lack of organic material and low levels of contaminants might point to low cultivation inputs. The positive controls using oxamyl with a simulated soil sample suggested that the method could detect retroactive molecules in soil and the results from the farm samples confirm that levels of contamination varied. The pattern was not entirely consistent, but greenhouse samples were always more toxic than control samples and air conditioned greenhouses often had more toxic soil eluates than normal greenhouses, presumably reflecting the increased resources, including agrochemicals used in the air conditioned units.

The contaminants from greenhouses on farm M clearly had significant amounts of toxic material in the soil and the dose-response experiments with diluted eluates produced dose-inhibition curves reminiscent of those seen with pure pesticides. It is interesting that the chemical analysis indicates that azinphos-methyl may be present in sample M2-2. This is an organophosphate pesticide with significant toxicity and environmental impact which has been banned in the EU since 2006 and was the subject of a law-suit by American Farm Workers [25]. Its effect on a range of biomarkers has been described and its contribution to environmental contamination assessed [26].

\section{Conclusions}

The studies included in this paper provide further evidence that pharyngeal pumping rate in C.elegans is a potentially useful biomarker assay for assessing the environmental impacts of soil toxicants. The assay described here has produced quantifiable responses to soil residues treated with standard doses of a widely used soil nematicide, as well as unknown toxicants eluted from soil samples collected in the field. The assay also has the additional benefits of being easy and cheap to set up using standard laboratory equipment and has the potential for use in environmental modelling in countries such as Saudi Arabia with developing agricultural economies where application of agrochemicals is not well controlled and where resources for chemical analysis are not readily available.

\section{Acknowledgements}

The authors wish to thank the University of Nottingham and the Promising Research Centre in Biological Control and Agriculture Information (BCARC) at Qassim University for support during the project.

\section{REFERENCES}

[1] Hamilton, D. J., Ambrus, Á., Dieterle, R. M., Felsot, A. S., Harris, C. A., Holland, P. T., Katayama, A., Kurihara, N., Linders, J., Unsworth, J. and Wong, S.-S. (2003). Regulatory limits for pesticide residues in water Pure Appl. Chem., 75: (8) 
$1123-1155$.

[2] International standards organisation ISO http://www.iso.org/iso/home/

[3] Kulkarni, Devdutt., Gergs, André., Hommen, Udo., Ratte, Hans, Toni. and Preuss Thomas G. (2013). A plea for the use of copepods in freshwater ecotoxicology. Environ Sci Pollut Res, 20: 75-85.

[4] Hoss S., Jansch S., Moser T., Junker T and Rombke J. (2009) Assessing the toxicity of contaminated soils using the nematode Caenorhabditis elegans as test organism. Ecotoxicology and Environmental Safety 72: 1811-1818.

[5] Rombke, J., Jansch, S., Junker,T., Pohl,B., Scheffczyk,A. and Schallnass,H.-J. (2006). Improvement of the applicability of ecotoxicological tests with earthworms, springtails, and plants for the assessment of metals in natural soils. Environ. Toxicol.Chem. 25,787.

[6] Hyman, L.H. (1951). The invertebrates: Acanthocephala, Aschelminthes, and Entopeocta. In: The pseudocoelomate bilatera, Vol III. Mc- Graw-Hill, New York.

[7] Andrassy, I., (1992). A short census of free-living nematodes. Fund. Appl. Nematol. 15, 187-188.

[8] Fiscus, D.A. and Neher, D.A., (2002). Distinguishing sensitivity of free-living soil nematode genera to physical and chemical disturbances. Ecol. Appl. 12: 565-575.

[9] Donkin, S. G. and Dusenbery, D. B. (1993). A soil toxicity test using the nematode Caenorhabditis elegans and an effective method of recovery. Arch. Environ. Contam. Toxicol.25: 145 .

[10] Power, M., van der Meer, J.R., Tchelet, R., Egli, T., Eggen, R., (1998). Molecular-based method can contribute to assessments of toxicological risks and bioremediation strategies. Journal of Microbiological Methods 32: 107-119.

[11] Freeman, M., N., Peredney, C.L. and Williams, P.L. (2000). A soil bioassay using the nematode Caenorhabditis elegans. In: Henshel, D.S.,Black, M.C.,Harrass, M.C. (Eds.),Environmental Toxicology and Risk Assessment :Standardization of Biomarkers for Endocrine Disruption and Environmental Assessment. American Society for Testing and Materials, West Conshohocken, PA, USA, pp. 305-318.

[12] Sochova, I., Hofman,J. and Holoubek, I. (2007). Effects of seven organic pollutants on soil nematode Caenorhabditis elegans. Environ.Int. 33: 798-804.

[13] Hoss, S., Arndt, M., Baumgarte, S., Tebbe,C., Nguyen-Thu, H. and Jehle, J. (2008). Effects of transgeniccorn and Cryl Ab protein on the nematode, Caenorhabditis elegans. Ecotoxicol. Environ. Saf. 70: 334-340.

[14] Anbalagan, C., Lafayette, I., Antoniou-Kourounioti, M., Haque, M., King, J., Johnsen, B., Baillied, D., Gutierrez, C., Rodriguez Martin JA and DE Pomerai D. (2012). Transgenic Nematodes As Biosensors For Metal Stress In Soil Pore
Water Samples. Ecotoxicology. 21: 439-55.

[15] Anbalagan, C., Lafayette, I., Antoniou-Kourounioti, M., Gutirrez, C., Martin, .JR., Chowdhuri, D.K. and DE Pomerai D. (2013). Use of transgenic GFP reporter strains of the nematode Caenorhabditis elegans to investigate the patterns of stress responses induced by pesticides and by organic extracts from agricultural soils. Ecotoxicology. 22: 72-85.

[16] Aschner, M., Leung, C. K. M., Williams, P. L., Benedetto, A., $\mathrm{Au}$, C., Helmcke, J.K. and Meyer, N.J. (2008). Caenorhabditis elegans: An Emerging Model in Biomedical and Environmental Toxicology. Toxicological Sciences 106(1): 5-28.

[17] Stringham-Durovic, E. G., and Candido, E. P. M. (1994). Transgenic hsp16- lacZ strains of the soil nematode Caenorhabditis elegans as biological monitors of environmental stress. Environmental Toxicology and Chemistry 13: 1211-1220.

[18] Boyd W.A. and Williams P.L. (2003). Comparison of the sensitivity of three nematode species to copper and their utility in aquatic and soil toxicity tests. Environ Toxicol Chem 22: 2768-2774.

[19] Avery L, and Horvitz,H.R., 1990 Effects of starving and neuroactive drugs on feeding in Caenorhabditis elegans J.Exp. Zool. 253:363-370

[20] Walkley, A and Black, I. A. (1934). An Examination of Degtjareff Method for Determining Soil Organic Matter and a Proposed Modification of the Chromic Acid Titration Method. Soil Sci. 37:29-37.

[21] Raizen, D. M. and Avery, L. (1994). Electrical activity and behavior in the pharynx of Caenorhabditis elegans. Neuron 12: 483-495.

[22] Hu, C., Dillon, J., Kearn, J., Murray, C. and O'Connor, V. (2013) NeuroChip: A Microfluidic Electrophysiological Device for Genetic and Chemical Biology Screening of Caenorhabditis elegans Adult and Larvae. PLoS ONE 8(5): e64297.

[23] Chuan, M.C., Shu, G.Y., and Liu, J.C. (1996). Solubility of heavy metals in a contaminated soil: Effects of redox potential and $\mathrm{pH}$. Water, Air, and Soil Pollution, Volume 90: Issue 3-4, pp 543-556.

[24] Khanna N, Cressman CP, Tatara CP, Williams PL. (1997). Tolerance of the nematode Caenorhabditis elegans to $\mathrm{pH}$, salinity, and hardness in aquatic media. Arch Environ Contam Toxicol; 32: 110- 4 .

[25] Earthjustice

http://earthjustice.org/sites/default/files/library/legal_docs/pe sticides-farmworkers-third-amended-complaint.pdf

[26] Schulz, R. (2004) Field studies on exposure, effects, and risk mitigation of aquatic nonpoint-source insecticide pollution : a review. J Environ Qual. 33(2):419-48. 